Int. J. Dev. Biol. 56: 909-918 (2012)

doi: $10.1387 / \mathrm{ijdb} .120135 \mathrm{gv}$

\title{
Human oocyte maturation in vitro
}

\author{
GIOVANNI COTICCHIO* MARIABEATRICE DAL-CANTO, MARIA-CRISTINA GUGLIELMO, \\ MARIO MIGNINI-RENZINI and RUBENS FADINI
}

Biogenesi, Reproductive Medicine Centre, Istituti Clinici Zucchi, Monza, Italy

\begin{abstract}
Oocytes from medium-sized antral follicles have already completed their growth phase and, if released from the follicular environment and cultured in vitro, are able to resume the meiotic process and mature. However, in vitro maturation (IVM) does not entirely support all the nuclear and cytoplasmic changes that occur physiologically as an effect of the ovulatory stimulus. Regardless, oocyte IVM is widely applied for the breeding of agriculturally important species. In assisted reproduction technology, IVM has been proposed as an alternative treatment to circumvent the drawbacks of standard ovarian stimulation regimens. Initially introduced to eliminate the risks of ovarian hyperstimulation syndrome afflicting women presenting with polycystic ovaries, subsequently IVM has been suggested to represent an additional approach suitable also for normovulatory patients. So far, in children born from IVM cycles, no doubts of an increased incidence of congenital abnormalities have been raised. Many more births would be achieved if novel IVM systems, currently dominated by empiricism, could be conceived according to more physiological criteria. Recent findings shedding new light on the control of meiotic progression, the support of cumulus cells to the oocyte cellular reorganization occurring during maturation, and the modulation of the stimulus that promotes oocyte maturation downstream the mid-cycle gonadotropin signal are likely to provide crucial hints for the development of more efficient IVM systems.
\end{abstract}

KEY WORDS: oocyte, meiosis, in vitro maturation, in vitro fertilization, assisted reproduction technology

\section{Introduction}

The development of a mature female gamete occurs through a lengthy and highly specialized process during which the cell massively increases in size and stockpiles molecules, organelles and spatial information that, following fertilization, will be crucial for the successful development of the embryo. After a period lasting from a few weeks to several months depending on the species, the oocyte of a preovulatory follicle has almost completed this process, and yet has not undergone a final series of molecular and cellular changes occurring during ovulation that are equally essential to attain full developmental competence. In particular, at ovulation over the course of several hours, the oocyte, arrested at the diplotene stage of meiotic prophase since the time of its formation in the fetal gonad, resumes meiosis and progresses to the metaphase stage of the second meiotic division (MII), while cytoplasmic organelles and macromolecules are reorganized in preparation for fertilization. In vivo, this concluding act of oogenesis, referred to as oocyte maturation, is triggered by mid-cycle luteinizing hormone (LH) surge and requires the co-ordinate participation of mural granulosa cells (MGC) and cumulus cells (CC). In vitro, upon release from a medium-large sized follicle, the oocyte is apparently capable to recapitulate the same process, as shown by the fact that in many cases it can resume meiosis, progress to MII and give rise to a zygote after fusion with the spermatozoon. In mammals, oocyte in vitro maturation (IVM) has been described as early as the thirties of the twentieth century (Pincus and Enzmann, 1935). Since then, it has been adopted as a tool to study under controlled, although artificial, conditions the final phases of oogenesis. It has also become a fundamental source of oocytes for the production of offspring of agriculturally valuable species through in vitro fertilization (IVF). In human assisted reproduction technology (ART), IVM has been proposed as an alternative intervention to minimize or eliminate the risks and drawbacks associated with intense stimulation of the ovary with gonadotropins. However, despite such an

Abbreviations used in this paper: ART, assisted reproductive technology; CC cumulus cell; COC, cumulus cell-oocyte complex; FSH, follicle stimulating hormone; hCG, human chorionic gonadotropin; IVF, in vitro fertilization; IVM, in vitro maturation; MGC, mural granulosa cell.

\footnotetext{
*Address correspondence to: Giovanni Coticchio. Biogenesi, Reproductive Medicine Centre, Istituti Clinici Zucchi, Via Zucchi 24, Monza, Italy. Tel/Fax: +39-039-838-3369. e-mail: coticchio.biogenesi@grupposandonato.it

Final, author-corrected PDF published online: 5 February 2013.
} 
interest, IVM methodology has remained largely empirical and, as a consequence, relatively inefficient. Current IVM protocols have changed little, if any, over several decades and are too simplistic, as an effect of lack of knowledge of the final and yet critical changes that transform a fully-grown primary oocyte into a mature gamete. Consistently, it should not surprise that across mammalian species the implantation and developmental potential of embryos derived from IVM oocytes has been reported to be significantly lower in comparison to in vivo matured oocytes. Regardless, IVM has continued to attract growing interest, especially in consideration of potential novel applications in human ART. This has driven the development of several lines of research whose results have led to a better understanding of the process of maturation. Findings of recent years have given us a much better perception of the ability of the oocyte to regulate the function of MGC and CC to achieve follicle growth and differentiation. We have also learned that the control of meiotic arrest throughout oogenesis and resumption at the beginning of maturation is fundamental to obtain a developmentally competent oocyte. Furthermore, the role of epidermal growth factor (EGF)-like proteins has emerged as a crucial element in the regulatory mechanism by which LH initiates oocyte maturation in vivo. These and other advances might lead to the development of more sophisticated IVM systems able to reproduce more physiologically and efficiently the process of oocyte maturation, thereby offering better opportunities for the application of IVM in biotechnology and reproductive biomedicine.

\section{Importance of IVM in research, animal breeding and human ART}

Current technology does not allow the direct observation of the oocyte in vivo. Manipulation of oogenesis in vivo has also limitations, as an effect of a myriad of systemic influences that can make the interpretation of individual experimental conditions difficult or equivocal. For these reasons, IVM has been conceived and developed as a more accessible investigation strategy to study the conclusive events of oogenesis. In fact, since the pioneering experience made with rabbit oocytes and reported by Pincus and Enzmann in 1935 (Pincus and Enzmann, 1935), IVM has been extended to a plethora of other mammalian species. Fully-grown oocytes enclosed in their vestment of CC can be isolated from antral follicles and matured in vitro after a culture period of 16-48 hours (depending on the species). This practice offers several important advantages, including the possibility to observe dynamically the process of maturation under a microscope and test the action of hormones, growth factors and biologically active molecules in a simplified system in which the confounding influences of the follicle, the ovary and the entire organism are eliminated (Eppig et al., 1988). More recently, the same reductionistic approach has been extended to the follicle as a functional unit, making possible the investigation of earlier stages of oogenesis in a similar fashion (Telfer and McLaughlin, 2011).

For study purposes, IVM was first established and brought to a certain level of success in rodent species (Schroeder and Eppig, 1984). In fact, the mouse and the rat have emerged as species particularly amenable to IVM, being easy to breed, manipulate and test for reproductive studies.

The boundaries of IVM have been progressively expanded to a number of other species for both scientific and commercial interests, including cow, pig, sheep, goat and horse. In particular, cow oocytes mature in vitro with a very high efficiency (over 90\%) providing an abundant and easily available source of material for the production of livestock through IVF and fuelling a flourishing agricultural industry. For example, it has been reported that, in a single year, over 130,000 cattle were produced worldwide from in vitro matured oocytes (Thibier, 2006).

Being a monovular species, the cow has also gained increasing importance as a more suitable model for the human, in whom IVM has been introduced to mitigate the unwanted implications of ART. In fact, the current strategy for IVF treatment involves the administration of high doses of gonadotropins to override the mechanism of follicle dominance and thereby achieve the growth, ideally up to the preovulatory stage, of several large follicles. Unfortunately, controlled ovarian stimulation involves various downsides, the most important of which is ovarian hyperstimulation syndrome (OHSS). In its severe form, OHSS is manifested by $2-5 \%$ of women subject to ovarian stimulation, require hospitalization and is potentially lifethreatening (Gomez et al., 2010). Women affected by polycystic ovary (PCO) and polycystic ovary syndrome (PCOS) are particularly predisposed to develop OHSS, as an effect of the growth of very numerous follicles even after the administration of relatively low doses of gonadotropins. IVM requires no or small doses of gonadotropins and does not imply the growth of follicles to a large size. Therefore it can totally abolish the risk of OHSS. PCO and PCOs patients have become the first natural candidates for IVM treatment as early as 1994 (Trounson et al., 1994). In more recent years, the range of clinical application of IVM has been extended to women with physiologically normal ovaries (usually defined as normovulatory) having appropriate characteristics (Fadini et al., 2009). In particular, it was shown that normovulatory women with high antral follicle count (Fadini et al., 2009) and elevated antiMüllerian hormone (AMH) levels (Fadini et al., 2011), traits that are suggestive of a good ovarian reserve, can be treated through IVM with significant chances of success. In the last few years, IVM has been also emphasized as an additional opportunity for female germ cell preservation in women suffering from cancer (Gidoni et al., 2008). Novel radio- and chemotherapies can significantly improve the prognosis of these patients (Mertens et al., 2001), but unfortunately imply major effects on ovarian function, often leading to premature ovarian failure. Technically, immature oocytes collected from antral follicles in the absence of gonadotropin administration may be cryopreserved before or after maturation in vitro (Cao et al., 2009). Therefore, IVM represents a suitable opportunity for the recovery of oocytes destined to cryopreservation in cases in which tumour estrogen-sensitivity and/or urgency to start therapy conflict with the implementation of a full controlled ovarian stimulation treatment (Wallace, 2011).

\section{Maturation in vivo and in vitro}

Maturation in vivo occurs during a very short temporal window if compared to the entire period of oogenesis, and yet has a critical importance for the attainment of a fully competent oocyte. Inhibition and induction of meiosis, physical and biochemical interaction with $\mathrm{CC}$, chromatin condensation, organelle redistribution and cytoskeletal changes are only a few example of phenomena taking place during maturation from which oocyte competence depends critically and that must be regulated in a precise and co-ordinated 
fashion. On another hand, maturation in vitro, intended as meiotic resumption and progression to the mature (MII) stage can often be achieved, but some of these and other phenomena may occur abnormally, compromising oocyte viability. This concept, better explained in the below examples, has been developed through experiments conducted especially in murine species and represents a paradigm that should inspire similar studies in other species.

\section{Meiotic arrest and progression}

A fundamental aspect of maturation is the control of meiosis progression. Fully-grown oocytes of mid-large antral follicles are competent to resume meiosis, undergo germinal vesicle breakdown (GVBD) and progress to MII following emission of the polar body I (PBI). Nevertheless, they are maintained in meiotic arrest until ovulation. The follicular environment is responsible for preventing premature meiotic resumption, as shown by the fact that cumulus cell-oocyte complexes (COCs) isolated from their follicles and cultured for an appropriate time mature spontaneously (Pincus and Enzmann, 1935). Endogenously produced cyclic adenosine monophosphate (cAMP) is the key element in this regulatory network, as shown in the mouse (Schultz et al., 1983). High levels of cAMP activate protein kinase A (PKA) (Bornslaeger et al., 1986) that, through a series of downstream effectors, in final analysis inhibits the protein complex CDK1/cyclin B complex (known also as meiosis-promoting factor, MPF), a positive regulator of meiotic progression (Jones, 2004). However, contrary to what initially thought, meiotic resumption in vitro does not occur as a consequence of an interruption of gap-junction-mediated cAMP transfer from MGC and CC to the oocyte. Firstly, CAMP is autonomously and constitutively produced by an oolemma-bound G-protein (Mehlmann et al., 2002). Secondly, upon isolation from the follicle, intraoocyte cAMP concentration drops because of activation of phosphodiesterase 3A (Cho et al., 1974). How this enzyme is regulated by signals derived from follicular cells has emerged recently. MGC and CC produce cyclic guanosine monophosphate (cGMP) via the membrane-bound guanylate cyclase NPR2, which is stimulated by the MGC-derived C-Type Natriuretic Peptide (CNP) (Zhang et al., 2010; Zhang et al., 2011). Once generated, cGMP diffuses into the oocyte via gap junctions that connect MGC, CC and the oocyte itself. In the oocyte, high concentrations of cGMP inhibit phosphodiesterase 3A-mediated degradation of cAMP, ensuring meiotic arrest (Vaccari et al., 2009). Inhibition of synthesis and transport of cGMP importantly contributes to meiotic resumption in vivo. In fact, convincing evidence suggests that mid-cycle LH surge induces a 20-fold reduction in intrafollicular cGMP levels (Norris et al., 2009) and closure of gap junctions connecting MGC and CC (Norris et al., 2008). Therefore, meiotic resumption in vitro may be explained by a disruption of transport of cGMP to the oocyte, as an effect of physical separation of the COC from MGC and the inability of surrounding CC to generate enough cGMP, being not exposed to follicular CNP. Under such conditions, intraoocyte cGMP decreases, phosphodiesterase 3Ainhibition is prevented, and finally cAMP is degraded. So, in a way, from the standpoint of cAMP/cGMP regulation, spontaneous maturation in vitro evokes what occurs in vivo, at least in part. However, it is arguable that this is sufficient to ensure a proper meiotic progression. Other mouse studies on the kinetics of meiosis in oocytes matured in vitro and in vivo are informative in this respect (Sanfins et al., 2004). By $2 \mathrm{~h}$ of culture, most IVM oocytes have already undergone GVBD, while by $5 \mathrm{~h}$ the progression to prometaphase stage occurs gradually. Differently, in in vivo matured oocytes, the germinal vesicles (GV) stage is still retained at $2 \mathrm{~h}$ from the application of an ovulatory stimulus and GVBD occurs only at $3 \mathrm{~h}$. Also, in vivo matured oocytes exhibit greater synchrony, as shown by the fact that the GV-prometaphase transition occurs in a much shorter time interval. An earlier meiotic resumption in IVM oocytes is presumably secondary to a precocious MPF activation, a circumstance that is strongly suspected to causes major perturbances of cytoskeletal rearrangements, such as the formation of large $\mathrm{MI}$ and MII spindles. In fact, GV oocytes treated transiently with roscovitin, an inhibitor of MPF, display a delay in GVBD and cytoskeletal characteristics more similar to in vivo matured oocytes (Sanfins et al., 2004). Consistently, it has been described in the pig (Marchal et al., 2001), cow (Ponderato et al., 2001) and horse (Franz et al., 2003) that temporary arrest at the GV stage with roscovitin at the beginning of the maturation period increases the developmental potential of IVM oocytes. The implications of these findings are least two-fold. Firstly, kinetics of meiosis may represent an important criterion to assess the regularity of the maturation process and can be significantly perturbed by spontaneous maturation in vitro. Secondly, the quality of IVM oocytes could be improved by culture strategies able to better control meiosis progression, in particular by delaying GVBD.

\section{Cumulus cell-oocyte contacts}

Cell-to-cell contacts between the oocyte, CC and MGC are another topical aspect of oocyte physiology with major implications for IVM. Throughout oogenesis, the oocyte and follicle cells form a functional syncytium via gap-junctional communications that

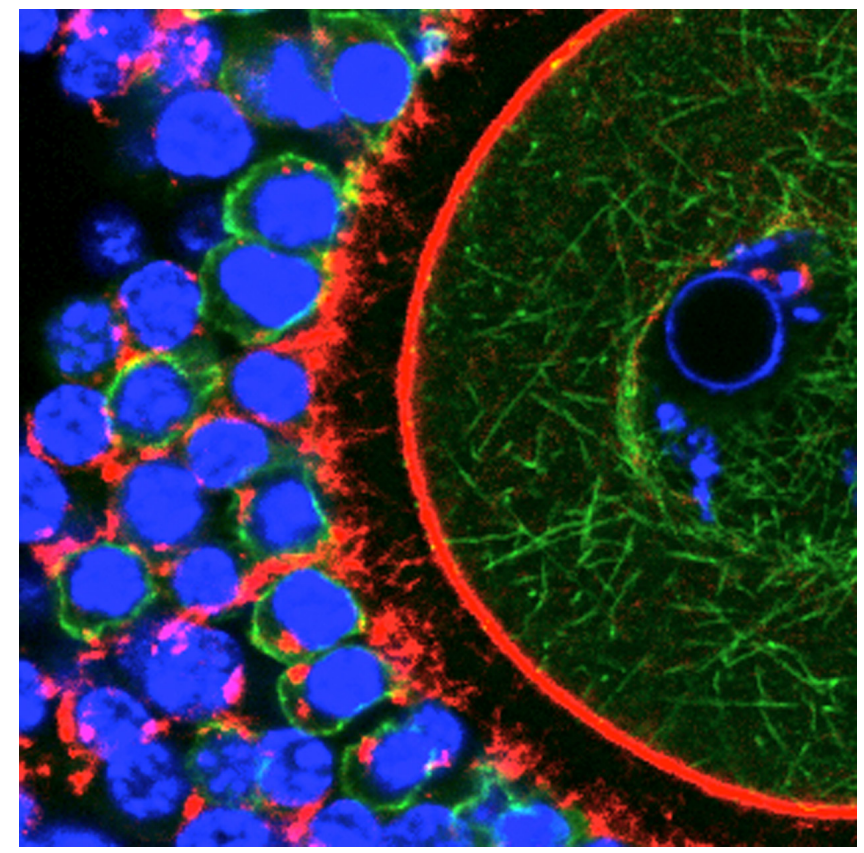

Fig. 1. Laser scanning confocal microscopy image illustrating a detail of a mouse immature cumulus cell-oocyte complex (COC). After isolation, the COC was fixed and stained for actin (red), DNA (blue) ad tubulin (green). Actin- rich transzonal projections are visible as thin filaments connecting cumulus cells to the oocyte. 
connect MGC with $\mathrm{CC}$ and the latter with the oocyte. Two types of connexins are involved in such a network, respectively cx43 and cx37. This ensures that small molecules essential for oocyte physiology (such as amino acids, cholesterol, and small regulative molecules) are supplied to the oocytes by the somatic compartment. The above described regulation of meiotic arrest and resumption operated by MGC- and CC-derived cGMP is a notable example of that. OocyteCC gap junctional communication requires not only a specialised type of intercellular contact, but also a remarkable CC phenotype. In fact, being the oocyte enclosed in the zona pellucida and physically compartmentalised from the rest of the follicle, surrounding CC must generate and guide through the zona a dense array of actin-rich axon-like protrusions, termed trans-zonal projections (TZPs), in order to make contact with the oolemma (Fig. 1), where gap junctions may be formed. Recent mouse experiments unveiled the specific importance of this highly specialized type of cell-to-cell contact that ensures not only gap-junctional communication, but also other regulatory actions of CC that profoundly influence oocyte cytoskeletal processes occurring during maturation. In mouse COCs that have been isolated from preovulatory follicles and analysed at different times after inducing maturation with human chorionic gonadotropin (hCG), TZPs arrangement can be observed in a very distinct pattern (Barrett and Albertini, 2010). At $0 \mathrm{~h}$ post-hCG they are densely distributed around the oocyte with only a moderate clustering in the portion of the oolemma where the germinal vesicle (GV) is cortically localized. At $2 \mathrm{~h}$, no major changes are observed, but in the 4-6 h interval TZPs organization changes dramatically. Following GVBD occurring within $2 \mathrm{~h}$ post-hCG and formation of the metaphase I (MI) spindle, which is assembled and remains anchored cortically, overall TZPs density is reduced around the whole oocyte surface, with the exception of the region where the spindle is localized. Therefore, under in vivo conditions, TZPs distribution is dynamically regulated and focalized around the portion of the oocyte cortex where the GV and the forming MI spindle are present. In vitro, in the absence of appropriate follicle stimulating hormone (FSH) supplementation, TZPs and oocyte cytoskeletal regulation are severely affected. Between 2 and $4 \mathrm{~h}$ of culture following isolation from preovulatory follicles, TZPs undergo a progressive reduction in number in comparison to the in vivo control and result undetectable by $6 \mathrm{~h}$. This is accompanied by disanchoring of the GV from the cortex by $2 \mathrm{~h}$. As a result of that, an abnormally large MI spindle is formed in a central position and migrates back to the cortex only at around $6 \mathrm{~h}$. Normal TZPs density and oocyte cytoskeletal dynamics are preserved if $\mathrm{FSH}$ is added to the culture system, but not if IVM is conducted in the presence of latrunculin, an actin-depolymerizing agent. Therefore, inappropriate IVM conditions appear to inhibit maintenance of TZPs around the oocyte, especially in the region where the GV and the forming spindle are anchored to the cortex. As a consequence, the GV loses its peripheral position and a large spindle is formed centrally, although is re-localized cortically afterwards. Formation of a large spindle is not without consequences, because it causes emission of a very large PBI (Sanfins et al., 2003) and, as a consequence of that, the loss of valuable cytoplasmic material that otherwise would contribute to the making of the embryo.

Such experiments teach us that manipulation of IVM conditions should be carefully considered because, if inadequate, it has the potential to perturb very fundamental processes, such as CC-oocyte interaction and spindle formation, thereby affecting oocyte developmental potential. These aspects of oocyte maturation should be

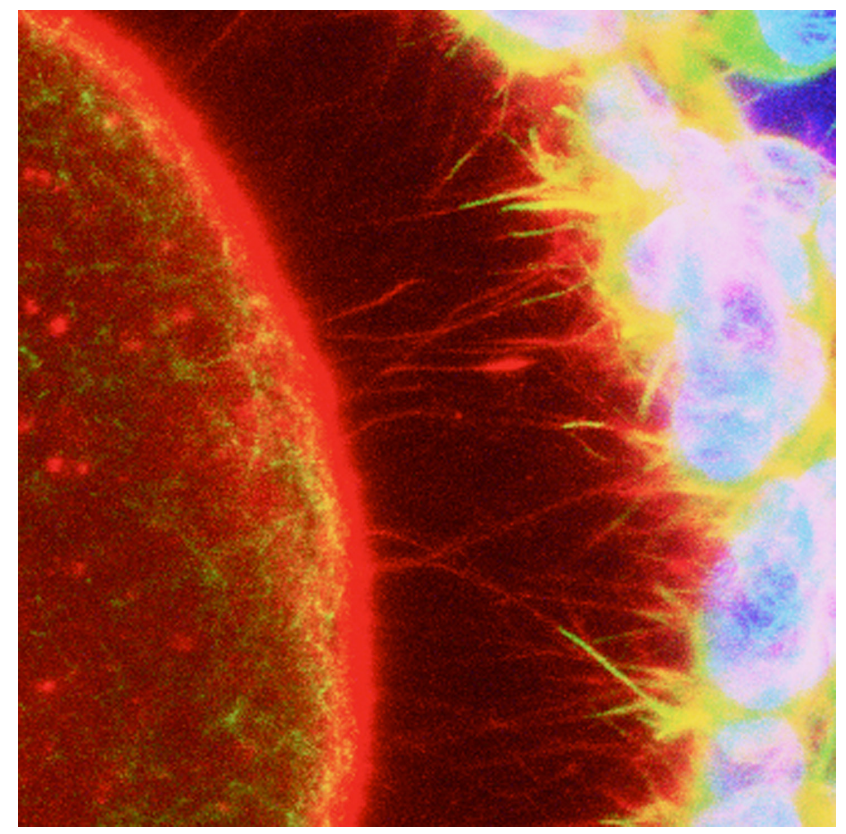

Fig. 2. Three-dimensional reconstruction of a detail of a human immature cumulus cell-oocyte complex. The cumulus cell-oocyte complex was fixed immediately after recovery and stained for actin (red), DNA (blue) ad tubulin (green), before laser scanning confocal microscopy analysis. The image was elaborated in order to emphasize the density of actin- rich transzonal projections connecting cumulus cells to the oocyte.

investigated also in the human species for their obvious clinical implications. No information has been gained on whether and how TZPs follow a precise dynamics during maturation and regulate GV positioning and spindle formation. In preliminary observations involving material derived from IVM cycles, we detected a very dense array of TZPs in COCs shortly after isolation from antral follicles (Fig. 2). However, due to the paucity of material donated for research, so far we have not been able to monitor the destiny of TZPs during the course of IVM and their possible influence on spindle formation. Premature loss of TZPs-mediated interactions is likely to affect other delicate aspects of maturation. For example, it is known that the ability of the oocyte to decondense the sperm head at fertilization is developed during maturation following accumulation of glutathione, a disulphide bond reducing agent derived from CC (Yoshida, 1993). Clearly, disruption of TZPs would prevent the transfer of glutathione from $\mathrm{CC}$ to the oocyte, with predictable consequences on fertilization.

\section{Endoplasmic reticulum and inositol 1,4,5-trisphosphate receptors}

In the mature oocyte, intracellular oscillations in the concentration of free calcium $\left(\mathrm{Ca}^{2+}\right)$ are pivotal to coordinate fertilization events, i.e. cortical granules release, resumption of meiosis, pronuclear formation, and recruitment of maternal RNAs (Ducibella etal., 2006). The ability to sustain intracellular $\mathrm{Ca}^{2+}$ oscillations is not entirely established in fully-grown GV-stage oocytes. Studies in the mouse established how regulation of intracellular $\mathrm{Ca}^{2+}$ is developed during maturation (Swann and Yu, 2008). $\mathrm{Ca}^{2+}$ is stored in the cisternae of the endoplasmic reticulum (ER). Atfertilization, release in the cytosol 
is mediated by inositol 1,4,5-trisphosphate $\left(\mathrm{IP}_{3}\right)$ generated from cleavage of the oolemma-bound lipid phosphatidyl-1,4-trisphosphate. Interaction of $\mathrm{IP}_{3}$ with its receptor situated in the membranes of the ER triggers the release of $\mathrm{Ca}^{2+}$ in the cytoplasm. ER redistribution and increase in $\mathrm{IP}_{3}$ receptor expression and sensitivity occurring during maturation in vivo are crucial to achieve maximal $\mathrm{Ca}^{2+}$ release ability in the mature oocyte. In the GV-stage oocyte, the ER is in continuity with the nuclear envelope and forms aggregate preferentially distributed in the oocyte interior (Mehlmann et al., 1995). At GVBD, ER membranes cluster around bundles of microtubules and envelope the newly formed $\mathrm{Ml}$ spindle. As maturation progresses, the ER cisternae and vesicles develop and form aggregates of 1-2 um occupying also the cortical cytoplasm, with a preferential accumulation in the vegetal hemisphere (opposite to the MII spindle position). In coincidence with ER growth and redistribution, the $\mathrm{IP}_{3}$ receptors increase in number and become localized in the oocytes cortex, area from which they were excluded in the immature oocyte (Mehlmann etal., 1996). These modifications of the ER and IP ${ }_{3}$ receptors are believed to account for the augmented sensitivity of mature oocytes to $\mathrm{Ca}^{2+}$-release mechanisms that regulate fertilization. Human oocytes display a similar pattern of changes in ER distribution and $\mathrm{IP}_{3}$ abundance (Mann et al., 2010). In GV-stage oocytes recovered from IVF cycles performed under a standard regimen (non-IVM) of ovarian stimulation, the ER is in most cases excluded from the cortical region and does not appear organized in clusters. Unlike the mouse, though, ER accumulation is not visible around the GV. In in-vivo matured oocytes, ER elements are associated in clusters of 2-3 um preferentially localized in the cortex, but are also discernible in more internal regions. Therefore, also in the human, during maturation the ER undergoes significant changes in its distributions that are associated to an enhanced ability to release $\mathrm{Ca}^{2+}$. These changes can be achieved in vitro. In fact, GV-stage oocytes derived from stimulated cycles and matured in vitro without cumulus cells display an array of cortical and more internal clusters similar to those typical of in-vivo matured oocytes. Conversely, in vitro maturation under the same conditions seems to affect the expression of the $\mathrm{IP}_{3}$ receptor, whose amount after maturation is lower in comparison to oocytes matured in vivo. This may cause a reduced sensitivity of in vitro matured oocytes to $\mathrm{Ca}^{2+}$-releasing mechanisms, as suggested by the observation that, following microinjection of $\mathrm{IP}_{3}$, the rise in intracellular free $\mathrm{Ca}^{2+}$ in in-vitro matured oocytes is comparable to the one of GV-stage, but not in vivo matured, oocytes. These experiments are very instructive because illustrate the dynamics of essential processes taking place during maturation and highlight possible divergences between maturation in vivo and in vitro. However, by no means they can be adopted to draw conclusions on the quality of in vitro matured oocytes generated in clinical IVM cycles. In fact, the human data reported above were generated from GV-stage oocytes derived from stimulated cycles, i.e. from material of notoriously compromised quality. In addition, IVM was conducted under rather inadequate conditions, especially considering the absence of surrounding CC that, as explained in the case of GV and spindle dynamics, are essential for the generation of a competent oocyte.

\section{Clinical regimens in human in vitro maturation}

Human IVF is generally perceived as a discipline emerged initially between the sixties and the seventies, mainly for the efforts of the Nobel prize R.G. Edwards (Edwards, 1965; Steptoe and Edwards,
1978). Indeed, Edwards is a giant of human ART and science in general. In reality, the very first steps of in vitro human conception were taken 30-40 years earlier. Amazed and inspired by Pincus and Enzmann who reported in 1935 the maturation in vitro and fertilization of oocytes of rabbit and other species (Pincus and Enzmann, 1935), in a Science manuscript dated 1944 John Rock and Miriam Menklin described the isolation of human oocytes from ovaries of gynaecological patients, their culture, fertilization and development into cleaving embryos (Rock and Menkin, 1944). Because these oocytes were retrieved between days 10 and 12 of an ovarian cycle, it is highly unlikely that they were already mature when were placed in culture. This circumstance, together with the fact that such oocytes were cultured in vitro for $22-28 \mathrm{~h}$ before insemination, suggests that the first human conceptuses generated in vitro derived from IVM oocytes.

\section{No gonadotropin priming}

In simple terms, the Rock and Menklin approach is the same that was originally adopted to introduce IVM in human ART in more recent times. In 1994, Trounson and colleagues described the first treatment of PCO patients (Trounson et al., 1994). As explained above, these women were selected on the basis of a large number (up to 30-40) of antral follicles present in their ovaries and the higher risk of OHSS associated to a standard ovarian stimulation treatment. No gonadotropins were administered and oocyte retrieval was scheduled when the larger follicle(s) was (were) smaller than 10-12 mm, stage at which dominance (in ovulation cycles) has not yet been established and smaller follicles have not been affected by phenomena of atresia. This scheme has survived virtually unchanged over the last decades and is still adopted in current practice also for the treatment of properly selected normovulatory patients (Fadini et al., 2009). It represents the most genuine form of IVM, in that no gonadotropins are delivered and all retrieved oocytes are immature. At recovery, COCs may display differences in the integrity and number of CC layers, but all oocytes are found invariably at the GV stage and are cultured for 30-48 h before assessing their meiotic status and proceeding with insemination, if appropriate. With few exceptions, in human IVM fertilization is achieved by intracytoplasmic sperm injection (ICSI), in consideration of mouse experiments showing that in IVM oocytes fertilization via standard IVF in attenuated by hardening of the zona pellucida (Schroeder and Eppig, 1984).

\section{Follicle stimulating hormone priming}

Alternative regimens have been introduced with the intent to improve oocyte yield and maturation rates in IVM cycles. Granulosa cells of antral follicles are responsive to FSH and it is conceivable that administration of even small doses of this hormone may improve the follicle health status and increase the number and quality of retrieved oocyte. Between 1999 and 2000, this approach was initially tested in normovulatory and PCO women with results not always consistent (Suikkari et al., 2000; Mikkelsen and Lindenberg, 2001). A more recent study does not seem to demonstrate a clear advantage in the delivery of FSH to normovulatory women (Fadini et al., 2009). Regardless, FSH priming remains an approach normally used in human IVM. In its more established version, it involves daily administration of 150 IU for 3 days starting from day 3 of 
cycle, followed by a period of coasting of 2-5 days in preparation of oocyte recovery (Mikkelsen and Lindenberg, 2001; Mikkelsen et al., 2003). This period of FSH withdrawal is part of the regimen for a precise reason. In fact, experiments in the cow showed that a short period of hormone deprivation after FSH priming improves oocyte quality by inducing mild atretic effects (Nivet et al., 2012). Atresia is usually considered a manifestation of follicle demise. However, early atresia reproduces events probably recognized by the oocyte as preovulatory signals, such as increase in progesterone and androgen synthesis and reduced metabolic support by granulosa cells. Perhaps, also in the human the full potential of FSH might be achieved by carefully dosing the effect of the coasting period, thereby inducing an appropriate atretic signal. From an oocyte perspective, the $\mathrm{FSH}$ priming scheme responds to the criteria of authentic IVM, because all retrieved COCs display a compact organization, include a GV stage oocyte and require a period of maturation before use.

\section{Human chorionic gonadotropin priming}

Bovine GV-stage oocytes can mature with rates of approximately $90 \%$, while their human counterparts rarely manifest maturation rates beyond $50-55 \%$. Per se, this represents a major limitation for the application of IVM in ART, irrespective of the quality of IVM oocytes. Therefore, human chorionic gonadotropin (hGC) has been suggested as an alternative form of gonadotropin priming to the aim of facilitating the resumption of meiosis in vivo before achieving full maturation in vitro (Chian et al., 1999; Chian et al., 2000). Introduced initially for the treatment of women with polycystic ovaries, this regimen has been subsequently extended also to normovulatory patients (Fadini et al., 2009). Usually, 10000 units of hCG are administered as soon as the largest follicle reaches a size of $10-12 \mathrm{~mm}$ and oocyte recovery is carried out 36-38 hours later. hGC treatment induces a major diversification in the cohort of retrieved COCs. Most COCs appear unaffected by the priming showing compact layers of cumulus and enclosing a GV-stage oocyte. This is not surprising, because most antral follicles available for recovery in an IVM cycle are small (4-10 mm). They express few, if any, LH receptors and therefore are unlikely to respond to the HCG signal (Hillier et al., 1995). However, approximately 30\% of COCs, derived probably from 10-13 mm follicles more responsive to hCG, display signs of full cumulus mass expansion (Dal Canto et al., 2012). The oocytes of these COCs may be found at different maturation stages, GV, GVBD or MII. This has several implications for an IVM cycle. Firstly, it introduces a semantic question. Under such conditions, to some extent IVM loses its true meaning because some oocytes are found mature at recovery and are used directly for treatment. Secondly, cycle efficiency clearly augments because such in vivo matured oocytes are expected to generate embryos with higher implantation rates. Thirdly, it imposes a diversification of culture requirements because COCs showing an expanded cumulus and presumably enclosing a mature oocyte are destined to short incubation of $4-6 \mathrm{~h}$ before insemination, while compact COCs that invariably are formed from a GV-stage oocyte are maintained in culture for at least $30 \mathrm{~h}$ in the attempt to achieve meiotic resumption and progression to MII. Furthemore, the retrieval of expanded COCs has another consequence. Despite $\mathrm{CC}$ are loosely connected and embedded in a highly hydrated and expanded extracellular matrix, it is difficult to discern precisely the oocyte meiotic status. Therefore, although after 4-6 hours of culture removal of CC in preparation for ICSI often reveals an MII oocyte, in many other cases oocyte maturation may be not accomplished. In case of immaturity, an extension of IVM for additional 24-30 h is problematic under such circumstances, considering that $\mathrm{CC}$, so important for the maturation process, have been removed to assess the oocyte meiotic status. This represents an unresolved problem of the hCG priming approach and objectively jeopardizes the quality of some oocytes.

\section{Follicle stimulating hormone/ human chorionic gonado- tropin (FSH/hCG) priming}

Chronologically, combined FSH and hCG priming has followed in more recent times the use of the two gonadotropins alone. The $\mathrm{FSH} / \mathrm{hCG}$ regimen involves usually daily administration of 150 IU for 3 days starting from day 3 of cycle, followed by delivery of $10000 \mathrm{IU}$ of hCG when the largest follicles reaches a size of 10$12 \mathrm{~mm}$. A recent study suggests that the FSH/hCG approach may represent the best option for normovulatory patients (Fadini et al., 2009). Likewise the case of hCG priming, success rates benefit from the possibility to utilize oocytes already mature at recovery, despite the persisting problems derived from the heterogeneity of the retrieved material.

\section{In vitro maturation systems}

In the report describing the first birth obtained by IVM oocytes retrieved from the ovaries of an IVM patient, maturation was conducted in 4-well plates using a non-specific medium (MEM) with addition of $\mathrm{FSH}$, LH and estradiol as hormones, in addition to maternal serum as supplements (Trounson et al., 1994). Incredibly, and disappointingly, such culture conditions have survived virtually unchanged until nowadays. Commercial IVM media have been introduced, but there is no evidence that their formulation is appropriate for oocyte maturation in vitro. In very few human IVM studies, if any, the relative impact of culture media (and their supplements) has been described. This is in striking contrast with the well-established notion that the type of IVM medium alone can largely influence the ability of mouse IVM oocytes to fertilize, develop to blastocyst and implant (van de Sandt et al., 1990). Clearly, comparison of multiple media appears very difficult in a human IVM context, in which material donated for research is extremely rare. However, every effort should be made to improve at least some basal conditions under which IVM is routinely performed.

$\mathrm{LH}$ is normally added to the culture mixture in view of its natural role as promoter of ovulation and oocyte maturation in vivo. Nevertheless, its use in vitro may be considered rather arbitrary because recent evidence indicated that, similar to the case of the mouse, human CC express very few LH receptors, especially in the case of small-medium sized follicles that are targeted in IVM cycles (Maman et al., 2012). So, LH supplementation might be irrelevant. In some IVM protocols, assuming that $\mathrm{CC}$ can respond to $\mathrm{LH}, \mathrm{hCG}$ is used as a substitute. However, such a choice does not consider that $\mathrm{LH}$ and hCG are not identical, the latter eliciting a different cell response, as shown by recent gene expression studies (Segers et al., 2012).

Contrary to the case of $\mathrm{LH}$, collective evidence suggests that FSH should be included in an IVM culture system. FSH is in fact 
generally recognized as a hormone that improves follicle cells health status (Hillier et al., 1995). However, no proof is available on the fact that the concentration normally adopted in IVM media, approximately $10^{-1} \mathrm{IU} / \mathrm{ml}$, is appropriate. Recent experiments in the cow suggest that the dose of FSH can profoundly influence COC function in vitro. In particular, it has been observed that a relatively high concentration $\left(10^{-1} \mathrm{lU} / \mathrm{ml}\right)$ of the hormone is unable to assure the maintenance of CC-oocyte gap junction communication. Vice versa, a much lower concentration $\left(10^{-4} \mathrm{IU} / \mathrm{ml}\right)$ preserves gap junction coupling. In such a way, at meiotic resumption chromatin condensation and transcription silencing can occur in a co-ordinated fashion, a condition required for the generation of a developmentally competent oocyte (Luciano et al., 2011).

Several other aspects of the culture systems employed in human IVM are still afflicted by empiricism. For examples, maternal serum has been, and still is, used as a protein supplement in IVM media, based on animal studies suggesting that serum improves overall oocyte quality. In reality, there is no evidence that serum is essential for an IVM system. Rather, in some cases, human IVM laboratories shifted from serum to human serum albumin or defined protein mixtures without observing an obvious decrease in their results (Dal Canto et al., 2006; Dal Canto et al., 2012). So, the use of serum should be discouraged also because, being an undefined mixture of proteins, lipids, hormones, grow factors and other biologically active molecules, represents a hurdle to the standardization of the IVM procedure.

Similarly, other facets of IVM systems are affected by subjectivity. Following recovery, $\mathrm{COC}$ are cultured, individually or in small groups, in 500 ul of medium contained in a 4-well plate. Under these conditions, possible factors released by $\mathrm{CC}$ and having a role in supporting oocyte maturation would be diluted down to very low concentrations. No data are known on the effect of culturing COCs in small volumes, using for examples microdrops under oil.

Another point that remains unexplored in human IVM concerns the composition of the atmosphere in which culture is carried out. Until a few years ago, most laboratory were equipped with incubators using $5 \% \mathrm{CO}_{2}$ in atmospheric air as gas environment. Recent reports seem to converge towards the notion that $\mathrm{O}_{2}$ should be used at a more physiological level, i.e. $5 \%$ instead of $20 \%$, especially for systems adopted for blastocyst culture (Waldenström et al., 2009). This has generated a debate on whether also IVM should be carried out in a low oxygen atmosphere (Banwell et al., 2007).

The above examples are instructive of the fact that culture systems used in human IVM often rely on principles that are not sustained by objective evidence, have not been developed further over almost 20 years and sometimes, as in the case of LH supplementation, do not necessarily reflect the physiology of the process of maturation. The improvement of IVM systems will represent a priority for future generations of reproductive biologists and embryologists.

\section{Developmental competence of in vitro matured human oocytes}

Introduced in the mid-nineties, clinical IVM has gained increasing interest especially in the last few years, but remains an approach that only few clinics currently apply on a routine basis. The reasons are manifold. Firstly, PCO and PCOS women -i.e. those in whom IVM can be implemented more appropriately - amount to 5-10\% of all ART patients (Gomez et al., 2010). Secondly, only a small fraction of normovulatory women display a relatively high number of antral follicles at the beginning of the ovarian cycle and, therefore, are eligible for IVM treatment (Fadini et al., 2009). Thirdly, collective evidence indicates that in vitro matured oocytes have a reduced developmental potential in comparison to their in vivo counterparts.

Concerning the competence of IVM oocytes, a major and still unresolved problem is represented by low maturation rates. Irrespective of whether gonadotropin priming is adopted, oocytes retrieved at the GV stage mature with a rate that rarely exceed 50-55\% (Mikkelsen and Lindenberg, 2001; Fadini et al., 2009). This frequency is considerably lower than the proportion of oocytes found at the MII stage in a standard ovarian stimulation cycle (approximately $80 \%$ ). So, IVM is affected by a net loss of material from the very beginning of the procedure, with obvious implications in terms of efficacy. On the contrary, IVM does not seem to reduce oocyte ability to support normal fertilization. In fact, similar to ovarian stimulation cycles, fertilization rates of $70-75 \%$ are normally achieved in IVM treatments (Fadini et al., 2009; Dal Canto et al., 2012). As previously discussed, ICSI is the almost universally applied method of insemination, although it has been demonstrated that fertilization can be achieved also by standard IVF (Söderström-Anttila et al., 2005). Embryos derived from IVM cycles are transferred usually on day 2 or day 3 post-insemination. In PCO/PCOS patients, pregnancy rates of $20-30 \%$ were reported since the early years of IVM history (Chian et al., 2000; Tan and Child, 2002). However, these frequencies were achieved to the cost of the transfer of a high number (3-5) of embryos, a sign that implantation rates were low in those cycles. In a recent retrospective case-control study involving patients with polycystic ovaries (Gremeau et al., 2012), Gremeau and colleagues were able to achieve a pregnancy rate of almost $20 \%$ by transferring much less embryos (on average 1.9), although in the same work it was also reported that implantation and pregnancy rates achieved in the same typology of patients was much higher in standard ovarian stimulation cases.

In a prospective randomized trial conducted in normovulatory

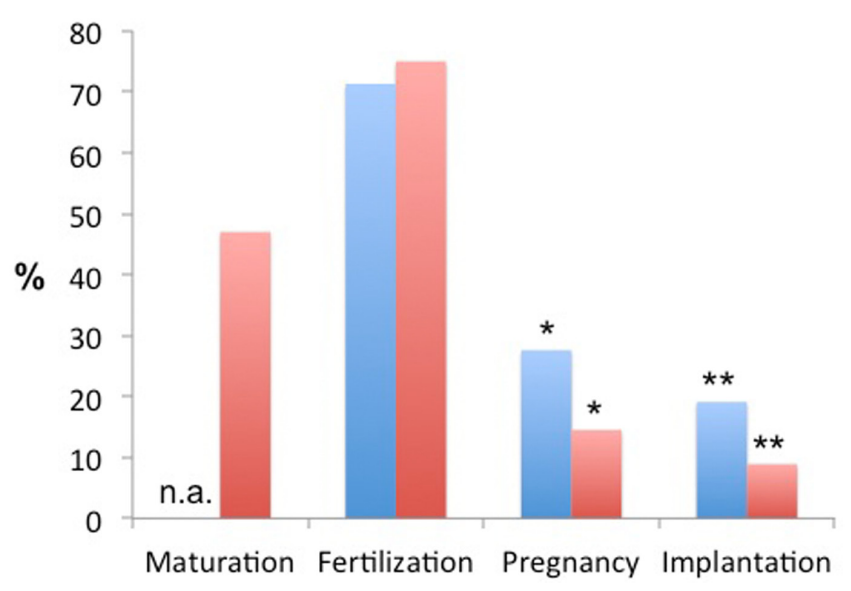

Fig. 3. Maturation, fertilization and developmental competence (expressed as pregnancy and implantation rates) of human oocytes derived from in vitro maturation cycles and matured in vivo (blue bars) or in vitro (red bars). Percentages are cumulative frequencies. See also text for further details (Dal Canto et al., 2012). ${ }^{*} p<0.0001,{ }^{*} p<0.0001$. 
women, we assessed the impact of different priming regimens (no priming, hCG, FSH, and FSH/hCG) on clinical outcome (Fadini et al., 2009). We found that the combined FSH/hCG priming generated significantly higher clinical pregnancy and implantation rates (26.5\% and $16.4 \%$, respectively), by transferring on average 1.81.9 embryos. Importantly, data from the same study also suggest that across the different IVM priming regimens in vitro matured oocytes undergo fertilization with unaltered rates but implant with frequencies that are clearly reduced in comparison to oocytes found mature at recovery in hCG-primed cycles (Fig. 3). This appears as an unquestionable proof that developmental competence is not fully expressed in IVM oocytes.

\section{The endometrium: the neglected organ}

In IVM cycles, oocytes are recovered as soon as the largest follicle reaches a size of $10-12 \mathrm{~mm}$, i.e. well before ovulation. Starting from the day of recovery, estradiol and progesterone are administered to support endometrial maturation. However, it is possible that the limited time intervening between oocyte recovery and embryo transfer is insufficient to allow full completion of the endometrial proliferative phase, a circumstance that might compromise the formation of a secretory endometrium and the chances of the transferred embryos to implant. Until recently, this question has been overlooked. Interestingly, however, novel findings suggest that the relatively lower clinical efficiency that characterise IVM might derive not exclusively from reduced oocyte quality caused by inadequate IVM conditions, but also from insufficient endometrial receptivity. Previous studies had shown that embryos generated from in vitro matured oocytes can be successfully cryopreserved and that high implantation rates can be achieved in recipients receiving embryos derived from donated IVM oocytes (Son et al., 2009). On this basis, De Vos and colleagues (De Vos et al., 2011) adopted an innovative strategy in IVM treatments. In patients primed with FSH only, they chose to cryopreserve and transfer in replacement cycles embryos derived from IVM oocytes. Interestingly, implantation and pregnancy rates were much higher in comparison to cycles in which embryos obtained after IVM were transferred fresh. If larger analyses will confirm such data, our perception of oocyte IVM will change. The imperative of improving IVM system will persist for the reasons discussed above, but the notion that IVM oocytes are intrinsically less developmentally competent will need to be reconsidered, giving relatively more emphasis to inadequate endometrial receptivity.

\section{Health of in vitro maturation children}

During IVM, the genetic and cytoplasmic integrity of the female germ cell might be compromised by not fully optimized manipulation conditions (Barrett and Albertini, 2007; Barrett and Albertini, 2010). This is consistent with experience matured in the cow, in which pregnancies from in-vitro matured oocytes and in-vitro produced embryos may be affected by increased fetal and placental weight, or aberrant development of fetal skeletal muscles and placental vessels (Farin et al., 2006). It is possible that these manifestations derive from deregulation during IVM of imprinted genes controlling fetal and placental growth (Li, 2002). On another hand, the long term health of mice generated from in vitro matured oocytes appears unaffected (Eppig et al., 2008). Doubts that IVM could introduce additional hazards in human ART have remained unaddressed, mainly because most of the estimated 2500 births derived from IVM cycles have not been reported in published studies or appropriately described. A study published some years ago on this subject involved 55 IVM, 217 IVF, and 160 ICSI babies (Buckett et al., 2007). In these births, odds ratio risk of congenital abnormalities in comparison to spontaneously conceived children was 1.42, 1.21, and 1.69, respectively. Other studies published on IVM children included even smaller numbers, overall indicating comparable health status to control groups, although with sporadic cases of major and minor abnormalities (Cha et al., 2005; Mikkelsen 2005; Soderstrom-Anttila et al., 2006). In a recent report concerning almost 200 babies born from IVM treatment, we did not detect major congenital abnormalities (Fadini et al., 2012), confirming previous evidence.

\section{Towards more physiological and efficient in vitro matu- ration systems}

Notions emerged in the last several years have drastically changed our perception of the complexity of the process of oocyte maturation. Basically, now we know that spontaneous maturation can occur in vitro, but does not recapitulate precisely all the events that make an oocyte competent. We have also learned that the oocyte represents the "centre of gravity" of the follicle. As such, it releases factors of the TGF- $\beta$ family (growth differentiation factors 9, GDF9, and bone morphogenetic factor 15, BMP15) able to divert CC from a general default pathway that characterize MGC to a condition that is largely oriented to meet the metabolic and intercellular communication needs of the oocyte (Su et al., 2007; Sugiura et al., 2008). Finally, we have appreciated that, in the microcosm of the COC, LH arguably plays a role, but rather its effects are mediated by (EGF)-like proteins (amphiregulin, epiregulin and betacellulin) (Park 2004). Clearly, future advances will depend on our ability to integrate all these elements in the IVM equation. We will need to learn how to control meiotic resumption and support a well-ordered process of chromatin condensation, preventing an abrupt and non-physiological drop in gap junction communication. We will be required to develop treatments able to preserve traits of the cumulus cell phenotype essential to the process of maturation (e.g. ability to produce cGMP under the action of a physiological stimulation) that might be lost during culture in vitro. Finally, we will have to design tools suitable to mimic the signal(s) that promote maturation in vivo, probably identifying appropriate doses of EGFlike proteins used at appropriate times. Recently, in the mouse and the cow, the simulated physiological oocyte maturation (SPOM) strategy has attempted to achieve at least part of these goals with results that, if confirmed in the human, might sign a turning point in human IVF (Albuz et al., 2010).

\section{Conclusion}

Clinical application of oocyte IVM has been brought up to the attention of the scientific community approximately 20 years ago. Since then, it has been adopted for an increasingly larger population of patients requiring ART treatment, PCO/PCOS and normovulatory subjects and women requiring fertility preservation in the absence of gonadotropin stimulation. IVM efficiency has remained lower in comparison to that of established ovarian stimulation regimens. 
Recent advances in the understanding of the regulation of meiosis and mutual interaction occurring of between the oocyte, CC and MGC promise to bring crucial innovations in IVM systems and, thereby, improve treatment standards.

\section{References}

ALBUZ, F.K., SASSEVILLE, M., LANE, M., ARMSTRONG, D.T., THOMPSON, J.G. GILCHRIST, R.B. (2010) Simulated physiological oocyte maturation (SPOM): a novel in vitro maturation system that substantially improves embryo yield and pregnancy outcomes. Hum. Reprod. 25: 2999-3011.

BANWELL, K.M., LANE, M., RUSSELL, D.L., KIND, K.L., THOMPSON, J.G. (2007) Oxygen concentration during mouse oocyte in vitro maturation affects embryo and fetal development. Hum. Reprod. 22: 2768-2775.

BARRETT, S.L., ALBERTINI, D.F. (2007) Allocation of gamma-tubulin between oocyte cortex and meiotic spindle influences asymmetric cytokinesis in the mouse oocyte. Biol. Reprod. 76: 949-957.

BARRETT, S.L., ALBERTINI, D.F. (2010) Cumulus cell contact during oocyte maturation in mice regulates meiotic spindle positioning and enhances developmental competence. J. Assist. Reprod. Genet. 27: 29-39.

BORNSLAEGER, E.A., MATTEI, P., SCHULTZ, R.M. (1986) Involvement of CAMPdependent protein kinase and protein phosphorylation in regulation of mouse oocyte maturation. Dev. Biol. 114: 453-462.

BUCKETT, W.M., CHIAN, R.-C., HOLZER, H., DEAN, N., USHER, R., TAN, S.L. (2007) Obstetric outcomes and congenital abnormalities after in vitro maturation, in vitro fertilization, and intracytoplasmic sperm injection. Obstet. Gynecol. 110: 885-891.

CAO, Y., XING, Q., ZHANG, Z.-G., WEI, Z.-L., ZHOU, P., CONG, L. (2009) Cryopreservation of immature and in-vitro matured human oocytes by vitrification. Reprod. Biomed. Online 19: 369-373.

CHA, K., CHUNG, H., LEE, D., KWON, H., CHUNG, M., PARK, L., CHOI, D., YOON, T. (2005) Obstetric outcome of patients with polycystic ovary syndrome treated by in vitro maturation and in vitro fertilization-embryo transfer. Fertil. Steril. 83: 1461-1465.

CHIAN, R.C., BUCKETT, W.M., TOO, L.L., TAN, S.L. (1999) Pregnancies resulting from in vitro matured oocytes retrieved from patients with polycystic ovary syndrome after priming with human chorionic gonadotropin. Fertil. Steril. 72:639-642.

CHIAN, R.C., BUCKETT, W.M., TULANDI, T., TAN, S.L. (2000) Prospective randomized study of human chorionic gonadotrophin priming before immature oocyte retrieval from unstimulated women with polycystic ovarian syndrome. Hum. Reprod. (Oxford, England) 15: 165-170.

CHO, W.K., STERN, S., BIGGERS, J.D. (1974) Inhibitory effect of dibutyryl cAMP on mouse oocyte maturation in vitro. J. Exp. Zool. 187: 383-386.

DAL CANTO, M., BRAMBILLASCA, F., MIGNINI RENZINI, M., COTICCHIO, G., MEROLA, M., LAIN, M., DE PONTI, E., FADINI, R. (2012) Cumulus cell-oocyte complexes retrieved from antral follicles in IVM cycles: relationship between COCs morphology, gonadotropin priming and clinical outcome. J. Assist. Reprod. Genet. 29: 513-519.

DAL CANTO, M., MIGNINI RENZINI, M., BRAMBILLASCA, F., CEPPARO, H., COMI, R., VILLA, A., et al.,2006) IVM--the first choice for IVF in Italy. Reprod. Biomed. Online 13: 159-165.

DE VOS, M., ORTEGA-HREPICH, C., ALBUZ, F.K., GUZMAN, L., POLYZOS, N.P., SMITZ, J., DEVROEY, P. (2011) Clinical outcome of non-hCG-primed oocyte in vitro maturation treatment in patients with polycystic ovaries and polycystic ovary syndrome. Fertil. Steril. 96: 860-864.

DUCIBELLA, T., SCHULTZ, R., OZIL, J. (2006) Role of calcium signals in early development. Sem. Cell and Dev. Biol. 17: 324-332.

EDWARDS, R.G. (1965) Maturation in vitro of human ovarian oöcytes. Lancet 2: 926-929.

EPPIG, J.J., DANIEL, S.A., SCHROEDER, A.C. (1988) Growth and development of mouse oocytes in vitro. Ann. N.Y. Acad. Sci. 541: 205-210.

EPPIG, J.J., O'BRIEN, M.J., WIGGLESWORTH, K., NICHOLSON, A., ZHANG, W., KING, B.A. (2008) Effect of in vitro maturation of mouse oocytes on the health and lifespan of adult offspring. Hum. Reprod. 24: 922-928.

FADINI, R., COMI, R., MIGNINI RENZINI, M., COTICCHIO, G., CRIPPA, M., DE PONTI, E., DAL CANTO, M. (2011) Anti-mullerian hormone as a predictive marker for the selection of women for oocyte in vitro maturation treatment. J. Assist. Reprod. Genet. 28: 501-508.

FADINI, R., DAL CANTO, M.B., MIGNINI RENZINI, M., BRAMBILLASCA, F., COMI, R., FUMAGALLI, D., LAIN, M., MEROLA, M., MILANI, R., DE PONTI, E. (2009) Effect of different gonadotrophin priming on IVM of oocytes from women with normal ovaries: a prospective randomized study. Reprod. Biomed. Online 19: 343-351.

FADINI, R., DAL CANTO, M.B., RENZINI, M.M., BRAMBILLASCA, F., COMI, R., FUMAGALLI, D., LAIN, M., DE PONTI, E. (2009) Predictive factors in in-vitro maturation in unstimulated women with normal ovaries. Reprod. Biomed. Online 18: 251-261.

FADINI, R., MIGNINI RENZINI, M., GUARNIERI, T., DAL CANTO, M., DE PONTI, E., SUTCLIFFE, A., SHEVLIN, M., COMI, R., COTICCHIO, G. (2012) Comparison of the obstetric and perinatal outcomes of children conceived from in vitro or in vivo matured oocytes in in vitro maturation treatments with births from conventional ICSI cycles. Hum. Reprod. 27: 3601-3608.

FARIN, P.W., PIEDRAHITA, J.A., FARIN, C.E. (2006) Errors in development of fetuses and placentas from in vitro-produced bovine embryos. Theriogenology 65: 178-191.

FRANZ, L.C., CHOI, Y.H., SQUIRES, E.L., SEIDEL, G.E., HINRICHS, K. (2003) Effects of roscovitine on maintenance of the germinal vesicle in horse oocytes, subsequent nuclear maturation, and cleavage rates after intracytoplasmic sperm injection. Reproduction 125: 693-700.

GIDONI, Y.S., TAKEFMAN, J., HOLZER, H.E.G., ELIZUR, S.E., SON, W.-Y., CHIAN, R.-C., TAN, S.L. (2008) Cryopreservation of a mother's oocytes for possible future use by her daughter with Turner syndrome: case report. Fertil. Steril. 90:2008.e9-12.

GOMEZ, R., SOARES, S.R., BUSSO, C., GARCIA-VELASCO, J.A., SIMÓN, C., PELLICER, A. (2010) Physiology and pathology of ovarian hyperstimulation syndrome. Sem. Reprod. Med. 28: 448-457.

GREMEAU, A.-S., ANDREADIS, N., FATUM, M., CRAIG, J., TURNER, K., MCVEIGH E., CHILD, T. (2012) In vitro maturation or in vitro fertilization for women with polycystic ovaries? A case-control study of 194 treatment cycles. Fertil. Steril. 98: 355-360.

HILLIER, S.G., SMYTH, C.D., WHITELAW, P.F., MIRÓ, F., HOWLES, C.M. (1995) Gonadotrophin control of follicular function. Hormone Res. 43: 216-223.

JONES, K.T. (2004) Turning it on and off: M-phase promoting factor during meiotic maturation and fertilization. Molecular Hum. Reprod. 10: 1-5.

LI, E. (2002) Chromatin modification and epigenetic reprogramming in mammalian development. Nature Rev. Genet. 3: 662-673.

LUCIANO, A.M., FRANCIOSI, F., MODINA, S.C., LODDE, V. (2011) Gap JunctionMediated Communications Regulate Chromatin Remodeling During Bovine Oocyte Growth and Differentiation Through cAMP-Dependent Mechanism(s) Biol. Reprod. 85: 1252-1259.

MAMAN, E., YUNG, Y., KEDEM, A., YERUSHALMI, G.M., KONOPNICKI, S., COHEN, B., DOR, J., HOURVITZ, A. (2012) High expression of luteinizing hormone receptors messenger RNA by human cumulus granulosa cells is in correlation with decreased fertilization. Fertil. Steril. 97: 592-598.

MANN, J.S., LOWTHER, K.M., MEHLMANN, L.M. (2010) Reorganization of the Endoplasmic Reticulum and Development of $\mathrm{Ca} 2+$ Release Mechanisms During Meiotic Maturation of Human Oocytes. Biol. Reprod. 83: 578-583.

MARCHAL, R., TOMANEK, M., TERQUI, M., MERMILLOD, P. (2001) Effects of cell cycle dependent kinases inhibitor on nuclear and cytoplasmic maturation of porcine oocytes. Molec. Reprod. Dev. 60: 65-73.

MEHLMANN, L.M., JONES, T.L.Z., JAFFE, L.A. (2002) Meiotic arrest in the mouse follicle maintained by a Gs protein in the oocyte. Science 297: 1343-1345.

MEHLMANN, L.M., MIKOSHIBA, K., KLINE, D. (1996) Redistribution and increase in cortical inositol 1,4,5-trisphosphate receptors after meiotic maturation of the mouse oocyte. Dev. Biol. 180: 489-498.

MEHLMANN, L.M., TERASAKI, M., JAFFE, L.A., KLINE, D. (1995) Reorganization of the endoplasmic reticulum during meiotic maturation of the mouse oocyte. Dev. Biol. 170: 607-615.

MERTENS, A.C., YASUI, Y., NEGLIA, J.P., POTTER, J.D., NESBIT, M.E., RUCCIONE, K., SMITHSON, W.A., ROBISON, L.L. (2001) Late mortality experience in five-year survivors of childhood and adolescent cancer: the Childhood Cancer Survivor Study. J. Clin. Oncol. 19: 3163-3172.

MIKKELSEN, A.L. (2005) Strategies in human in-vitro maturation and their clinica outcome. Reprod. Biomed. Online 10: 593-599. 
MIKKELSEN, A.L., HØST, E., BLAABJERG, J., LINDENBERG, S. (2003) Time interval between $\mathrm{FSH}$ priming and aspiration of immature human oocytes for in-vitro maturation: a prospective randomized study. Reprod. Biomed. Online 6: 416-420.

MIKKELSEN, A.L., LINDENBERG, S. (2001) Benefit of FSH priming of women with PCOS to the in vitro maturation procedure and the outcome: a randomized prospective study. Reproduction 122: 587-592.

NIVET, A.-L., BUNEL, A., LABRECQUE, R., BELANGER, J., VIGNEAULT, C., BLONDIN, P., SIRARD, M.-A. (2012) FSH withdrawal improves developmental competence of oocytes in the bovine model. Reproduction 143: 165-171.

NORRIS, R.P., FREUDZON, M., MEHLMANN, L.M., COWAN, A.E., SIMON, A.M., PAUL, D.L., LAMPE, P.D., JAFFE, L.A. (2008) Luteinizing hormone causes MAP kinase-dependent phosphorylation and closure of connexin 43 gap junctions in mouse ovarian follicles: one of two paths to meiotic resumption. Development 135: 3229-3238.

NORRIS, R.P., RATZAN, W.J., FREUDZON, M., MEHLMANN, L.M., KRALL, J., MOVSESIAN, M.A., WANG, H., KE, H., NIKOLAEV, V.O., JAFFE, L.A. (2009) Cyclic GMP from the surrounding somatic cells regulates cyclic AMP and meiosis in the mouse oocyte. Development 136: 1869-1878.

PARK, J.Y. (2004) EGF-Like Growth Factors As Mediators of LH Action in the Ovulatory Follicle. Science 303: 682-684.

PINCUS, G., ENZMANN, E.V. (1935) The comparative behavior of mammalian eggs in vivo and in vitro: I. The activation of mammalian eggs. J. Exp. Med. 62: 665-675.

PONDERATO, N., LAGUTINA, I., CROTTI, G., TURINI, P., GALLI, C., LAZZARI, G. (2001) Bovine oocytes treated prior to in vitro maturation with a combination of butyrolactone I and roscovitine at low doses maintain a normal developmental capacity. Molec. Reprod. Dev. 60: 579-585.

ROCK, J., MENKIN, M.F. (1944) In vitro fertilization and cleavage of human ovarian eggs. Science 100: 105-107.

SANFINS, A., PLANCHA, C.E., OVERSTROM, E.W., ALBERTINI, D.F. (2004) Meiotic spindle morphogenesis in in vivo and in vitro matured mouse oocytes: insights into the relationship between nuclear and cytoplasmic quality. Hum. Reprod. 19: 2889-2899.

SANFINS, A., LEE, G.Y., PLANCHA, C.E., OVERSTROM, E.W., ALBERTINI, D.F. (2003) Distinctions in meiotic spindle structure and assembly during in vitro and in vivo maturation of mouse oocytes. Biol. Reprod. 69: 2059-2067.

SCHROEDER, A.C., EPPIG, J.J. (1984) The developmental capacity of mouse oocytes that matured spontaneously in vitro is normal. Dev. Biol. 102: 493-497.

SCHULTZ, R.M., MONTGOMERY, R.R., BELANOFF, J.R. (1983) Regulation of mouse oocyte meiotic maturation: implication of a decrease in oocyte cAMP and protein dephosphorylation in commitment to resume meiosis. Dev. Biol. 97: 264-273.

SEGERS, I., ADRIAENSSENS, T., WATHLET, S., SMITZ, J. (2012) Gene expression differences induced by equimolar low doses of LH or hCG in combination with FSH in cultured mouse antral follicles. J. Endocrinol. 215: 269-280.

SON, W.-Y., CHUNG, J.-T., GIDONI, Y., HOLZER, H., LEVIN, D., CHIAN, R.-C., TAN, S.L. (2009) Comparison of survival rate of cleavage stage embryos produced from in vitro maturation cycles after slow freezing and after vitrification. Fertil. Steril. 92: 956-958.

SODERSTROM-ANTTILA, V. SALOKORPI, T., PIHLAJA, M., SERENIUS-SIRVE, S., SUIKKARI, A.-M. (2006) Obstetric and perinatal outcome and preliminary results of development of children born after in vitro maturation of oocytes. Hum. Reprod. 21: 1508-1513.
SÖDERSTRÖM-ANTTILA, V., MÄKINEN, S., TUURI, T., SUIKKARI, A.-M. (2005) Favourable pregnancy results with insemination of in vitro matured oocytes from unstimulated patients. Hum. Reprod. (Oxford, England) 20: 1534-1540.

STEPTOE, P.C., EDWARDS, R.G. (1978) Birth after the reimplantation of a human embryo. Lancet 2: 366

SU, Y.Q., SUGIURA, K., WIGGLESWORTH, K., O'BRIEN, M.J., AFFOURTIT, J.P., PANGAS, S.A., MATZUK, M.M., EPPIG, J.J. (2007) Oocyte regulation of metabolic cooperativity between mouse cumulus cells and oocytes: BMP15 and GDF9 control cholesterol biosynthesis in cumulus cells. Development 135: 111-121.

SUGIURA, K., SU, Y.Q., DIAZ, F.J., PANGAS, S.A., SHARMA, S., WIGGLESWORTH, K., O'BRIEN, M.J., MATZUK, M.M., SHIMASAKI, S., EPPIG, J.J. (2008) Oocytederived BMP15 and FGFs cooperate to promote glycolysis in cumulus cells. Development 135: 786-786.

SUIKKARI, A.M., TULPPALA, M., TUURI, T., HOVATTA, O., BARNES, F. (2000) Lutea phase start of low-dose FSH priming of follicles results in an efficient recovery, maturation and fertilization of immature human oocytes. Hum. Reprod. (Oxford, England) 15: 747-751.

SWANN, K., YU, Y. (2008) The dynamics of calcium oscillations that activate mammalian eggs. Int. J. Dev. Biol. 52: 585-594.

TAN, S.L., CHILD, T.J. (2002) In-vitro maturation of oocytes from unstimulated polycystic ovaries. Reprod. Biomed. Online 4 Suppl 1: 18-23.

TELFER, E., MCLAUGHLIN, M. (2011) In vitro Development of Ovarian Follicles. Sem. Reprod. Med. 29: 015-023.

THIBIER, M. (2006) Transfers of both in vivo-derived and in vitro-produced embryos in cattle still on the rise and contrasted trends in other species in 2005. IETS Newsletter 12-18.

TROUNSON, A., WOOD, C., KAUSCHE, A. (1994) In vitro maturation and the fertilization and developmental competence of oocytes recovered from untreated polycystic ovarian patients. Fertil. Steril. 62: 353-362.

VACCARI, S., WEEKS, J.L., HSIEH, M., MENNITI, F.S., CONTI, M. (2009) Cyclic GMP signaling is involved in the luteinizing hormone-dependent meiotic maturation of mouse oocytes. Biol. Reprod. 81: 595-604.

VAN DE SANDT, J.J., SCHROEDER, A.C., EPPIG, J.J. (1990) Culture media for mouse oocyte maturation affect subsequent embryonic development. Molec. Reprod. Dev. 25: 164-171.

WALDENSTRÖM, U., ENGSTRÖM, A.-B., HELLBERG, D., NILSSON, S. (2009) Low-oxygen compared with high-oxygen atmosphere in blastocyst culture, a prospective randomized study. Fertil. Steril. 91: 2461-2465.

WALLACE, W.H.B. (2011) Oncofertility and preservation of reproductive capacity in children and young adults. Cancer 117: 2301-2310.

YOSHIDA, M. (1993) Role of glutathione in the maturation and fertilization of pig oocytes in vitro. Molec. Reprod. Dev. 35: 76-81.

ZHANG, M., SU, Y.Q., SUGIURA, K., WIGGLESWORTH, K., XIA, G., EPPIG, J.J. (2011) Estradiol Promotes and Maintains Cumulus Cell Expression of Natriuretic Peptide Receptor 2 (NPR2) and Meiotic Arrest in Mouse Oocytes In Vitro. Endocrinology 152: 4377-4385

ZHANG, M., SU, Y.Q., SUGIURA, K., XIA, G., EPPIG, J.J. (2010) Granulosa Cell Ligand NPPC and Its Receptor NPR2 Maintain Meiotic Arrest in Mouse Oocytes. Science 330: 366-369. 


\section{Further Related Reading, published previously in the Int. J. Dev. Biol.}

Effect of genistein alone and in combination with okadaic acid on the cell cycle resumption of mouse oocytes A Van Cauwenberge and $\mathrm{H}$ Alexandre Int. J. Dev. Biol. (2000) 44: 409-420

Developmental competence of immature pig oocytes under the influence of EGF, IGF-I, follicular fluid and gonadotropins during IVM-IVF processes

M J Illera, P L Lorenzo, J C Illera and R M Petters

Int. J. Dev. Biol. (1998) 42: 1169-1172

Interphase-like chromatin configuration induced by cycloheximide in maturing pig oocytes: effects of protein phosphatase inhibitors

J Rozinek, J Petr, R Grocholová and F Jílek

Int. J. Dev. Biol. (1996) 40: 1171-117

Activation of in vitro matured mouse oocytes arrested at first or second meiotic metaphase Z Polanski

Int. J. Dev. Biol. (1995) 39: 1015-1020

Kinetics of MPF and histone $\mathrm{H} 1$ kinase activity differ during the G2- to M-phase transition in mouse oocytes

T Jung, R M Moor and J Fulka

Int. J. Dev. Biol. (1993) 37: 595-600

5 yr ISI Impact Factor $(2011)=2.959$

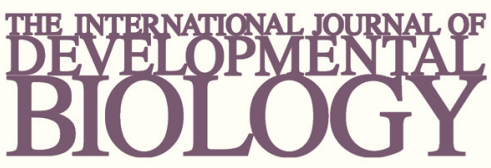

Volume 54 Nos. 6/7
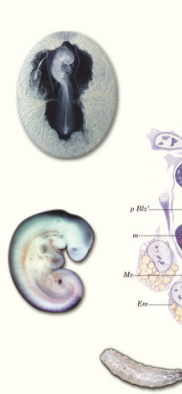

Developmental Hematopoiesis
Special Issue

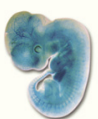

(2) (2) (2)

(3) (1)

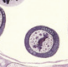

(2)
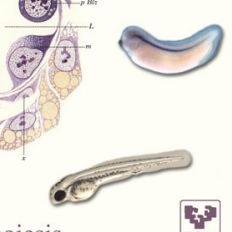

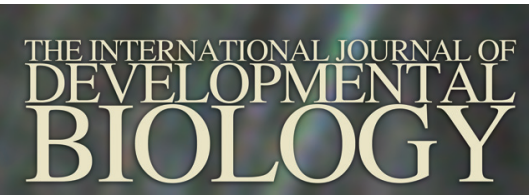

Volume 56 Nos. 1/2/3

Special Issue

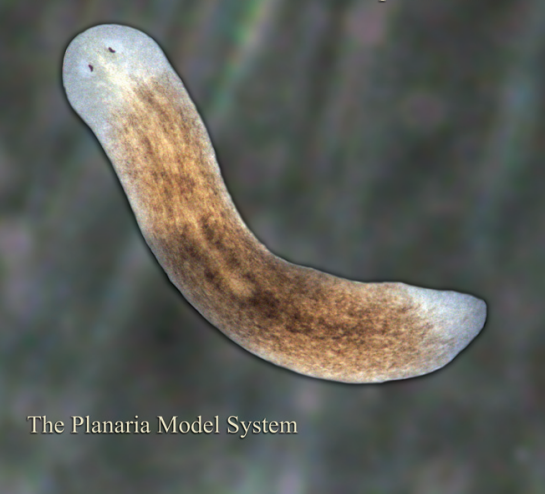

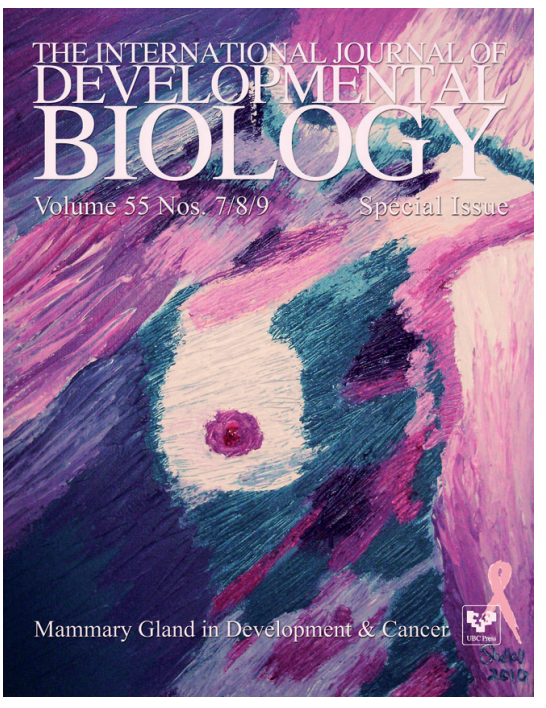

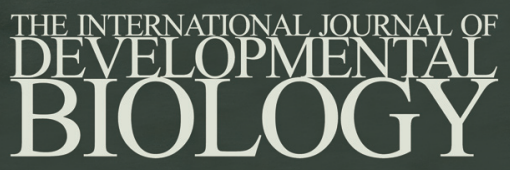

Volume 55 Nos. $4 / 5$

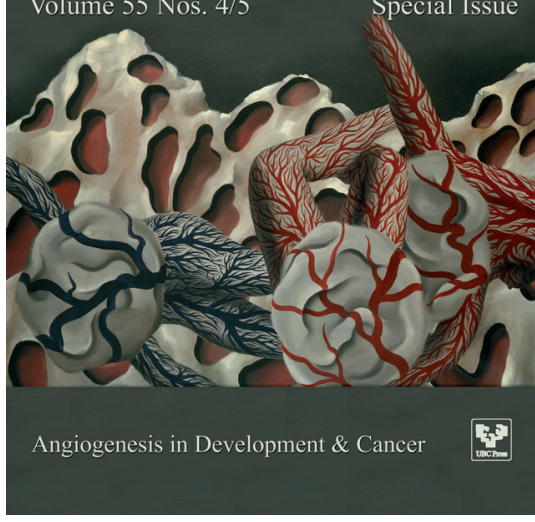

